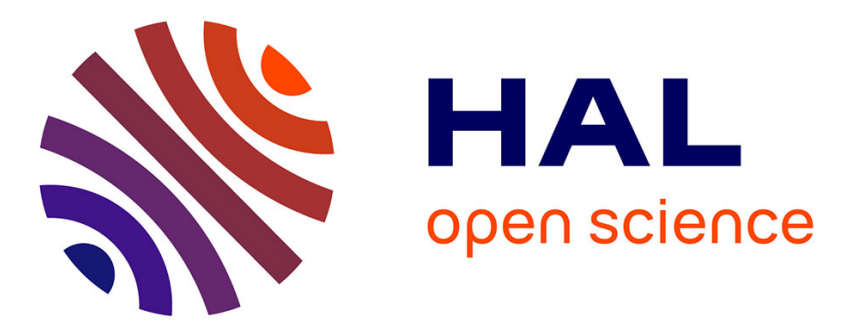

\title{
A Markov chain model for drop ratio on one-packet buffers DTNs
}

Victor Ramiro, Dinh-Khanh Dang, Gwilherm Baudic, Tanguy Pérennou, Emmanuel Lochin

\section{- To cite this version:}

Victor Ramiro, Dinh-Khanh Dang, Gwilherm Baudic, Tanguy Pérennou, Emmanuel Lochin. A Markov chain model for drop ratio on one-packet buffers DTNs. 2015 IEEE 16th International Symposium on "A World of Wireless, Mobile and Multimedia Networks" (WoWMoM), Jun 2015, Boston, United States. pp.1-6, 10.1109/WoWMoM.2015.7158190 . hal-02549742

\section{HAL Id: hal-02549742 \\ https://hal.science/hal-02549742}

Submitted on 21 Apr 2020

HAL is a multi-disciplinary open access archive for the deposit and dissemination of scientific research documents, whether they are published or not. The documents may come from teaching and research institutions in France or abroad, or from public or private research centers.
L'archive ouverte pluridisciplinaire HAL, est destinée au dépôt et à la diffusion de documents scientifiques de niveau recherche, publiés ou non, émanant des établissements d'enseignement et de recherche français ou étrangers, des laboratoires publics ou privés. 


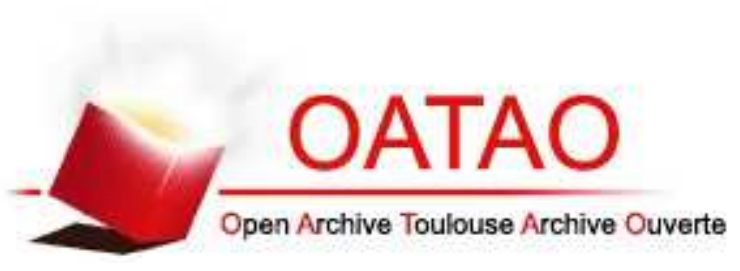

\section{Open Archive TOULOUSE Archive Ouverte (OATAO)}

OATAO is an open access repository that collects the work of Toulouse researchers and makes it freely available over the web where possible.

This is an author-deposited version published in : http://oatao.univ-toulouse.fr/ Eprints ID : 13910

To cite this version : Ramiro, Victor and Dang, Dinh Khanh and Baudic, Gwilherm and Pérennou, Tanguy and Lochin, Emmanuel A Markov chain model for drop ratio on one-packet buffers DTNs. In: IEEE WoWMoM Workshop on Autonomic and Opportunistic Communications (AOC), 14 June 2015 (Boston, United States)

Any correspondance concerning this service should be sent to the repository administrator: staff-oatao@listes-diff.inp-toulouse.fr 


\title{
A Markov chain model for drop ratio on one-packet buffers DTNs
}

\author{
Victor Ramiro*, Dinh-Khanh Dang*, Gwilherm Baudic*, Tanguy Pérennou*, Emmanuel Lochin* \\ *Université de Toulouse, ISAE-SUPAERO, France \\ $\{$ first.second $\} @$ isae.fr
}

\begin{abstract}
Most of the efforts to characterize DTN routing are focused on the trade-off between delivery ratio and delay. Buffer occupancy is usually not considered a problem and most of the related work assumes infinite buffers. In the present work, we focus on the drop ratio for message forwarding considering finite buffers. We model message drops with a continuous time Markov chain (CTMC). To the best of our knowledge, there is no previous work with such approach. We focus on the worst case with 1-packet buffers for message forwarding in homogeneous inter-contact times (ICT) and 2-class heterogeneous ICT. Our main contribution is to link the encounter rate(s) with the drop ratio. We show that the modeled drop ratio fits simulation results obtained with synthetic traces for both cases.
\end{abstract}

Keywords-DTN, Modeling, Characterization, Drop ratio, Continuous time Markov chain

\section{INTRODUCTION}

Delay Tolerant Networks (DTNs) are a well-known paradigm to allow communication between peers when infrastructure is scarce [1]. Communications are handled in a store-carry-and-forward format in DTNs. Hence, we benefit from peers communication opportunities (given by node movements, periodicity of contacts/connections, etc) to deliver data. Usually, it is not possible to establish a direct end-to-end path between source and destination; instead, a spatio-temporal path may be created by contact opportunities.

The DTNs pure definition based on the delay tolerance nature of communications covers a big spectrum of networks [2]: space communications, vehicular networks, sensor networks, opportunistic networks, etc. All these scenarios have in common to impose strong restrictions on the resources available to the nodes (energy, memory, processing power). Many works have focused on those different restrictions providing a better understanding in terms of characterization, mobility, energy consumption, or performance metrics such as delay, delivery or drop ratios to name a few.

A pioneering work in the field of DTN performance modeling was [3], where the authors provide a Markov model for the 2-hop and unrestricted multicopy routing protocols in the case of homogeneous exponentially distributed intercontact times (ICTs). Markov modeling was subsequently applied to Epidemic routing [4]-[6], Spray And Wait [7], [8] and Binary Spray and Wait [9]. Other modeling tools include Ordinary Differential Equations [10], [11] or Petri Nets [12].

Such works showed that unrestricted buffers and epidemic routing will provide the best delivery ratio [10], even though the number of message copies increases exponentially, as predicted by the SIR (Susceptible-Infected-Recovered) model
[13]. The buffer occupancy is usually not considered a problem, or one of less importance, to the point that most of the related work assumes infinite buffers [3]-[9], [14]-[17]. Heterogeneous ICTs, either for pairs [9], [15], [16] or groups [5], [14], [17] of nodes, are also rarely considered. A more detailed literature overview can be found in Table I.

We focus our work on a specific type of sensor network where mobile nodes perform a measurement and then transmit their data by message forwarding among their peers. Two groups of nodes are considered: sources which perform measurements, and destinations acting as collection points. A message can reach any collection point, as in an anycast network. We study the behavior of message forwarding in terms of number of dropped messages when we increase the number of messages generated in this sensor network. We use simple forwarding of messages to avoid including new message copies in the network (via replication), as this would only increase the probability of dropping a message.

We restrict our study to networks where nodes can carry only one message. Although it might seem limited, this case captures the situation of bigger buffers almost filled up with messages, to the point that every node has at most one free space in its buffer. Somehow this represents the basic limits of a DTN in terms of absorption capabilities: after reaching a given buffer occupancy, no more messages can be injected unless nodes start dropping messages due to buffer saturation.

In Section II we propose a continuous time Markov chain (CTMC) to characterize the drop of messages in 1-message buffers DTNs. The objective is to exhibit an upper bound to the drop ratio. From this, we can extract the trade-off between the number of sources and destinations nodes in order to achieve a desired drop ratio. We show that the outputs of the CTMC model fit to DTN simulation outputs (Section III). Our contribution is twofold:

1) We study a DTN network where nodes can store only one message in their buffer. Messages are forwarded from source to destination. Encounters between nodes are homogeneous. We model the drop ratio when increasing the number of messages (Section II-B), and provide simulations that match this dropping model (Sections III-A and III-B);

2) We extend our model to the case where nodes are divided among two groups with different encounter rates (Section II-C). We provide simulations to compare with the extended model (Section III-C). 
Table I: Previous literature considering finite/infinite buffers. Only [10] characterizes the drop ratio with finite buffers, using ODEs and focusing on homogeneous ICT and epidemic routing.

\begin{tabular}{|c|c|c|c|c|c|c|c|}
\hline \multirow{2}{*}{ Reference } & \multirow{2}{*}{ Buffer size } & \multirow{2}{*}{ Exponential parameters } & \multirow{2}{*}{ Routing protocols } & \multicolumn{4}{|c|}{ Performance metrics derived } \\
\hline & & & & Delay & Delivery ratio & Number of copies & Drop ratio \\
\hline [10] & Finite & Homogeneous & Epidemic & $\checkmark$ & - & $\checkmark$ & $\checkmark$ \\
\hline [11] & Finite & Homogeneous & Spray and Wait & $\checkmark$ & - & - & - \\
\hline [12] & Finite & Homogeneous & Epidemic, 2-hop & $\checkmark$ & $\checkmark$ & - & - \\
\hline [18] & Finite & Not mentioned & 2-hop & $\checkmark$ & - & - & - \\
\hline$[3]-[9],[14]-[17]$ & Infinite & - & - & $\checkmark$ & $\checkmark$ & $\checkmark$ & - \\
\hline This paper & Finite & $\begin{array}{c}\text { Homogeneous } \\
\text { Two-class Heterogeneous }\end{array}$ & Forwarding & - & - & - & $\checkmark$ \\
\hline
\end{tabular}

\section{Drop Message MODEL}

In this section, we define the basic notation and global hypotheses of the model. Then we introduce the specifics of our model for two cases: homogeneous intercontact time distribution and a restricted heterogeneous case with two classes of nodes.

\section{A. Model basics}

We consider a DTN with $N$ identical nodes $\mathcal{N}=$ $\{1,2,3, \ldots, N\}$ with a buffer capacity of one message. We consider $S<N$ message sources and $M$ initial copies of the same message. Notice that $S=M$ due to the buffer restriction. The $M$ messages are delivered to any of $D<N-S$ destinations. Unless stated differently, we consider $D=1$. Intermediary nodes act as forwarders of those $M$ messages. Hence, no extra copies are created in the evolution of the process. The goal is to determine the distribution of dropped messages and the drop ratio over time.

Let $0 \leq t_{i, j}(1) \leq t_{i, j}(2)<\ldots$ be the successive encounter times among nodes $i$ and $j$. We consider that the transmission time of a message is negligible with respect to the time it takes to two nodes to meet one another. It follows that the $n$-th intercontact time between $i$ and $j$ is $i c t_{i, j}(n)=t_{i, j}(n+1)-t_{i, j}(n)$. Later we set specific hypotheses on the nature of the processes $\left\{t_{i, j}\right\}$. Since each node can keep only one message, each time a contact occurs we try to forward it. Hence, when node $i$ and $j$ meet either: (i) only one of the nodes has a message, it is instantaneously transmitted, or (ii) both nodes have a message, then one is chosen at random to instantaneously transmit its message while the other drops its message.

\section{B. The $(d, c)$ model: homogeneous case}

For the homogeneous case, we assume that the processes $\left\{t_{i, j}(k), \quad k \geq 1, \forall i \neq j \in \mathcal{N}\right\}$ are mutually independent and homogeneous Poisson processes with rate $\lambda>0$. Hence the random variables $\left\{i c t_{i, j}(k), k \geq 1, \forall i \neq j \in \mathcal{N}\right\}$ are mutually independent and exponentially distributed with mean $1 / \lambda$.

To calculate the number of dropped messages, we introduce a continuous time Markov chain $(X(t), t>0)$. The states of the chain are $(d, c)$, where $d$ represents the number of dropped messages and $c$ the number of message copies. Our initial state is $(0, M)$. The transitions from a state $(d, c)$ are either to $(d, c-1)$ when a message is delivered or to $(d+1, c-1)$ when a drop occurs. The rate to encounter a destination will be $D \lambda$. Since we have $c$ nodes with a copy of the message, the former transition will happen with rate $c D \lambda$. The latter occurs with rate $\frac{c(c-1)}{2} \lambda$ given the number of combinations where two nodes with a message meet. In Figure 1, we detail the possible transitions for the general case. The absorbing states of the chain are in the form $(d, 0)$ with $0 \leq d \leq M-1$. Notice that when reaching the absorbing states, we must impose some border conditions. Since the last message will be delivered with probability 1 , we cannot transit from state $(d, 1)$ to $(d+1,0)$. In Figure 2, we draw the complete Markov chain for $M$.

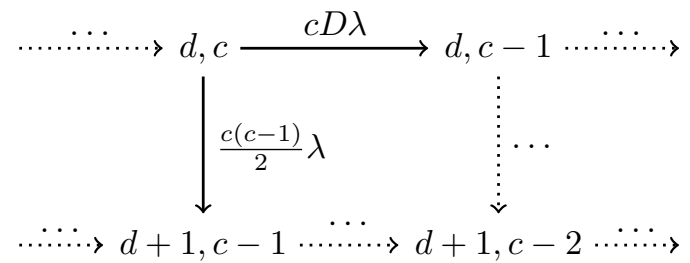

Figure 1: Chain transitions from a given state $(d, c)$. Either a destination is found among $D$ possibilities, leading to state $(d, c-1)$, or there is a drop, leading to $(d+1, c-1)$. State $(d+1, c-2)$ is included to show the progression of states.

We can easily calculate the probabilities of going out from the state $(d, c)$. Indeed, the embedded Markov chain for $X(t)$ allows to write the probabilities to jump between states as shown in (1).

$$
\begin{gathered}
P((d, c) \rightarrow(d, c-1))=\frac{c D \lambda}{c D \lambda+\frac{c(c-1)}{2} \lambda}=\frac{2 D}{c+2 D-1} \\
P((d, c) \rightarrow(d+1, c-1))=\frac{\frac{c(c-1)}{2} \lambda}{c D \lambda+\frac{c(c-1)}{2} \lambda}=\frac{c-1}{c+2 D-1}
\end{gathered}
$$

The absorbing states probabilities are defined as:

$$
P_{M}(d)=P\left(X_{\infty}=(d, 0) \mid X(0)=(0, M)\right), 0 \leq d \leq M-1
$$

Since the process is a feed-forward process, these probabilities can be easily calculated with a dynamic programming algorithm. The drop rate distribution is defined as the expected value of reaching the absorbing states over the number of starting messages $\frac{1}{M} \sum_{d=0}^{M-1} d P_{M}(d)$. 


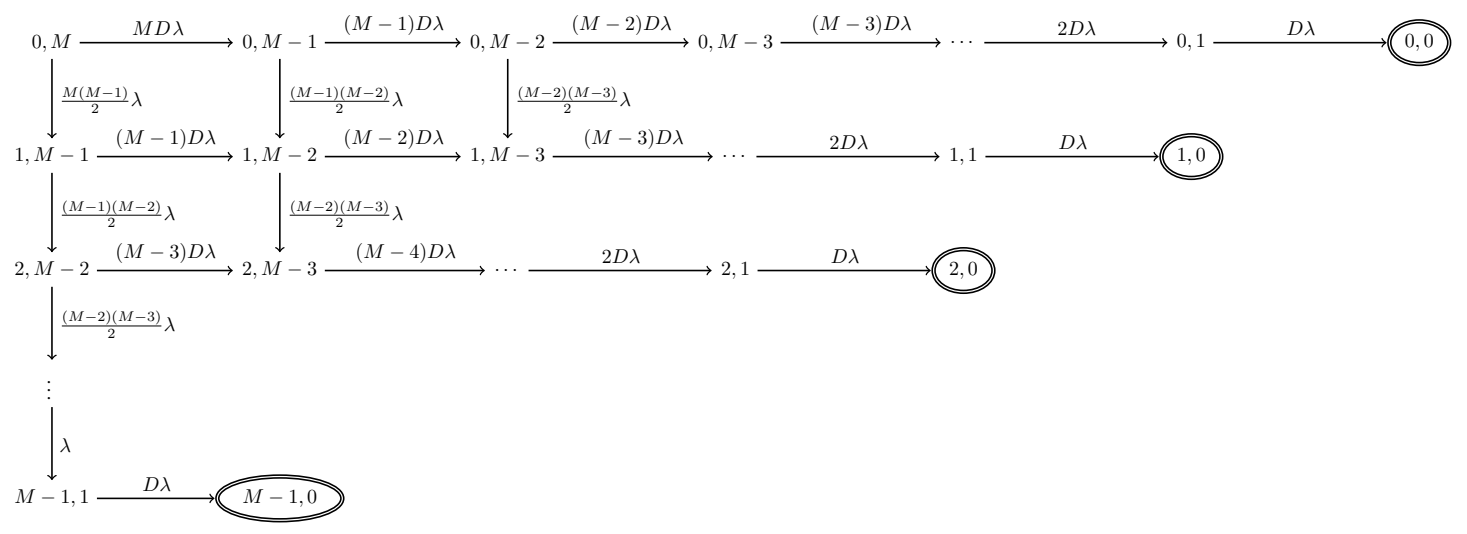

Figure 2: Complete Markov chain for the drop process. When there are no more messages to deliver, we reach a final state $(d, 0)$ with $d$ being the number of messages dropped.

It is important to notice that the probabilities are independent of the process arrival rate $\lambda$. Therefore, for any $\left\{t_{i, j}\right\}$ defined as before, we expect the same drop ratio results.

\section{The $\left(d, c_{1}, c_{2}\right)$ model: heterogeneity with two classes}

We extend the $(d, c)$ model to the case of two different classes of nodes $\mathcal{C}_{1}$ and $\mathcal{C}_{2}$, with $\left|\mathcal{C}_{1}\right|=N_{1}$ and $\left|\mathcal{C}_{2}\right|=N_{2}$ such that $N=N_{1}+N_{2}+D$. For convenience, we impose $D=1$. To keep the problem symmetrical, the destination does not belong to any class of nodes. We have $M=M_{1}+M_{2}$ messages distributed as $M_{1}$ in $\mathcal{C}_{1}$ and $M_{2}$ in $\mathcal{C}_{2}$.

The heterogeneity is defined such that the processes $\left\{t_{i, j}\right\}$ have different rates for each class: independent homogeneous Poisson processes with rate $\lambda_{1}$ in class $\mathcal{C}_{1}$ and with rate $\lambda_{2}$ in class $\mathcal{C}_{2}$, while inter-class interactions are given by independent and homogeneous Poisson processes with rate $\lambda$. As before, it follows that the random variables $i c t_{i, j}$ are mutually independent and exponentially distributed with the reciprocal of $\lambda_{1}, \lambda_{2}$ or $\lambda$ according to the case.

The differences between the values of $\lambda_{1}, \lambda_{2}$ and $\lambda$ define different speeds for the group encounter rates (and hence the waiting times between connections). For instance, this allows to model one group that will deliver its messages faster than the other. A faster delivery implies having more nodes with free buffers, hence reducing the probability of drops. The interclass communication allows to balance the drops allowing to pass messages from one class to another. From the symmetry of the problem, the destination meets nodes from $\mathcal{C}_{1}$ with rate $\lambda_{1}$ and nodes from $\mathcal{C}_{2}$ with rate $\lambda_{2}$. This restriction can easily be removed and does not affect the general result.

We model the states as $\left(d, c_{1}, c_{2}\right)$ where $d$ is the number of drops, $c_{1}$ is the number of copies in class $\mathcal{C}_{1}$ and $c_{2}$ is the number of copies in class $\mathcal{C}_{2}$. We identify three different kinds of transitions from the state $\left(d, c_{1}, c_{2}\right)$ :

1) Delivery transitions: we meet a destination with rates $\lambda_{i}$ in class $\mathcal{C}_{i}$. Since we have $c_{i}$ message copies it follows that the transitions rate are $c_{1} \lambda_{1}$ for $\left(d, c_{1}-\right.$ $\left.1, c_{2}\right)$ and $c_{2} \lambda_{2}$ for $\left(d, c_{1}, c_{2}-1\right)$;

2) Drop transitions: the number of combinations in class $\mathcal{C}_{i}$ where two nodes having a message meet is $\frac{c_{i}\left(c_{i}-1\right)}{2} \lambda_{i}$. Also we count the $c_{1} c_{2}$ combinations where two nodes from different classes having a message meet with rate $\lambda / 2^{1}$. It follows that the transition rates are $c_{1}\left(c_{1}-1\right) \lambda_{1} / 2+c_{1} c_{2} \lambda / 2$ for $\left(d+1, c_{1}-1, c_{2}\right)$ and $c_{2}\left(c_{2}-1\right) \lambda_{2} / 2+c_{1} c_{2} \lambda / 2$ for $\left(d+1, c_{1}, c_{2}-1\right)$

3) Inter-class transitions: in this case we count the number of combinations where a node from class $\mathcal{C}_{i}$ passes a message to a free node from class $\mathcal{C}_{j \neq i}$. This happens $c_{i}\left(N_{j}-c_{j}\right)$ times with rate $\lambda$. It follows that the transition rates are $c_{1}\left(N_{2}-c_{2}\right) \lambda$ for $\left(d, c_{1}-1, c_{2}+\right.$ $1)$ and $c_{2}\left(N_{1}-c_{1}\right) \lambda$ for $\left(d, c_{1}+1, c_{2}-1\right)$.

Figure 3 presents the general transitions from a state $\left(d, c_{1}, c_{2}\right)$ to all possible states described before. Same as before, we have $M$ absorbing states in the form $(d, 0,0)$ with $0 \leq d \leq M-1$. Notice that we arrive to the absorbing state either from $(d, 1,0)$ with rate $\lambda_{1}$ or from $(d, 0,1)$ with rate $\lambda_{2}$. Again, we need to rule out some transitions. For instance from $\left(d, N_{1}, c_{2}\right)$ we cannot go to $\left(d, N_{1}+1, c_{2}-1\right)$ or from $\left(d, 0, c_{2}\right)$ to $\left(d,-1, c_{2}+1\right)$. We do not present the complete chain due to the impossibility to draw it because of its size.

To calculate the dropping probabilities, we use the fact that the chain previously defined is an absorbing Markov chain. We do the following: $(i)$ enumerate the $N_{s}$ valid states of the chain $\left(d, c_{1}, c_{2}\right)$ with $0 \leq d \leq M-1,0 \leq c_{1} \leq N_{1}$ and $0 \leq c_{2} \leq$ $N_{2}$. This allows to define the mapping from $1 \leq i \leq N_{s}$ to state $s_{i}=\left(d^{\prime}, c_{1}^{\prime}, c_{2}^{\prime}\right)$ for all the valid states in the chain. (ii) define the matrix $A$ with coefficients $a_{i j}$ as the rate transform from state $s_{i}$ to state $s_{j}$ and the matrix $R$ with coefficients $r_{i j}$ as the rate from state $s_{i}$ to absorbing state $s_{j}^{*}$. Notice that the size of matrix $A$ is $N_{s} \times N_{s}$ and the matrix $R$ is $M \times N_{s}$. (iii) finally, we define $B=(I-A)^{-1} R$ where the $i^{t h}$ row is the distribution of absorbing states if the initial state is $s_{i}$. Since the absorbing states correspond to drop states, it follows that $B$ is the dropping distribution $P_{M}(d)$.

\footnotetext{
${ }^{1}$ We can split the inter-class Poisson processes into two counting processes with rate $\lambda p$ and $\lambda(1-p)$. The splitting probability corresponds to the drop probability when two nodes have a message. Since we take one at random, we have $\lambda p=\lambda(1-p)=\lambda / 2$.
} 


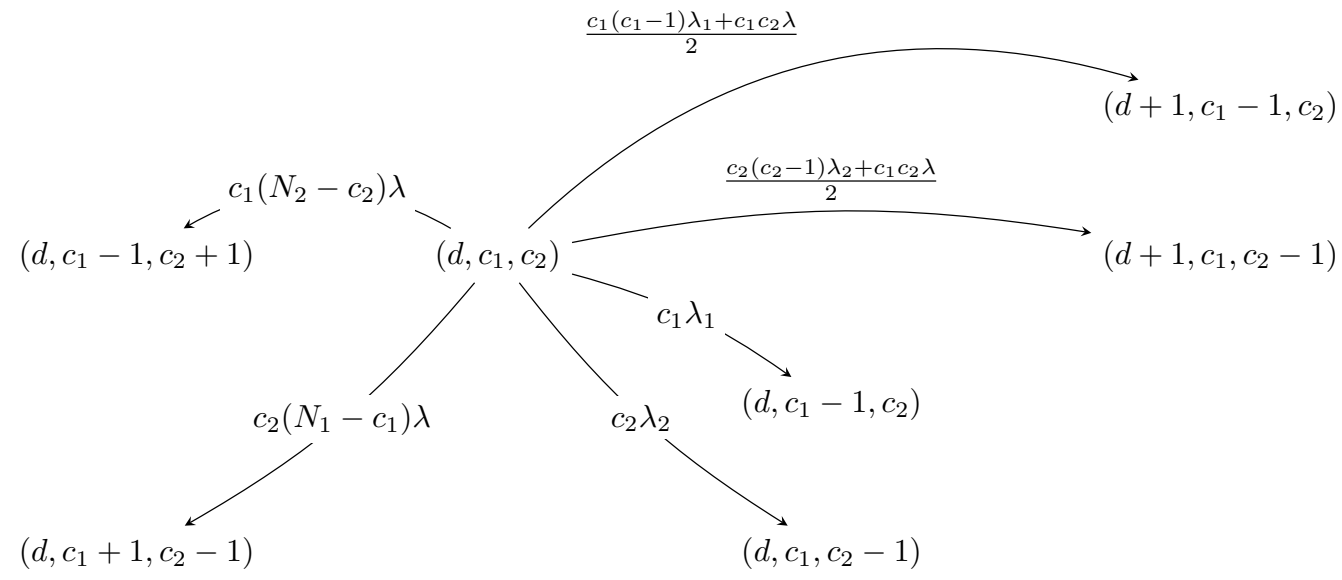

Figure 3: Chain transitions from a given state $\left(d, c_{1}, c_{2}\right)$. We observe the increase in number of states and transitions w.r.t the $(d, c)$ model shown in Figures 1 and 2: inter-class transitions $\left(d, c_{1}-1, c_{2}+1\right)$ and $\left(d, c_{1}+1, c_{2}-1\right)$; delivery transitions $\left(d, c_{1}-1, c_{2}\right)$ and $\left(d, c_{1}, c_{2}-1\right)$; and drop transitions $\left(d+1, c_{1}-1, c_{2}\right)$ and $\left(d+1, c_{1}, c_{2}-1\right)$.

\section{Simulation Setup}

In this section, we present the main results of the comparison between a simulated DTN network and the modeled CTMC results. We generate pairwise ICTs distributed as defined in Section II. We then run an event driven simulation to perform the forwarding of the messages in the network and calculate the drop ratio for a given configuration. We compare the simulated and predicted drop ratio for both $(d, c)$ and $\left(d, c_{1}, c_{2}\right)$ models. Table II presents an overview of the simulations we cover in this section. The model results have been obtained with MATLAB, while the continuous time simulations are implemented with $\mathrm{R}$.

We run all simulations with $N=100$ nodes and buffer size $B=1$. We repeat each simulation 10 times and provide the average results within a $95 \%$ confidence level. The network occupancy is defined as $\rho=S / N$. Sources are increased to represent the following percentages $\rho \in$ $\{1,2,5,10,15, \ldots, 80,95,98\}$.

\begin{tabular}{c|cccc} 
& $\rho$ & D & Model & Parameters \\
\hline E1 & $\nearrow$ & 1 & $(d, c)$ & $\lambda=500$ \\
E2 & $\nearrow$ & $\nearrow$ & $(d, c)$ & $\lambda=500$ \\
E3 & $\nearrow$ & 1 & $\left(d, c_{1}, c_{2}\right)$ & $\lambda_{1}=2.5, \lambda_{2}=200, \lambda=66.6$ \\
E4 & $\nearrow$ & 1 & $\left(d, c_{1}, c_{2}\right)$ & $\lambda_{1}^{\prime}=10, \lambda_{2}^{\prime}=100, \lambda^{\prime}=55$
\end{tabular}

Table II: Summary of simulations and their parameters $(\rho$ : network occupancy, D: destinations, $\nearrow$ : increasing).

\section{A. Homogeneous case: single destination}

As defined in Table II, we present the results of simulation $E 1$ with $\lambda=500$. As said in Section II, we assume that all pairs follow the homogeneous case with $\left\{t_{i, j} \sim\right.$ PoissonProcess $(\lambda)\}$ and $\left\{i c t_{i, j} \sim \exp \left(\lambda^{-1}\right)\right\}$.

Figure 4 shows how increasing the number of sources (hence the network occupancy) increases the drop ratio, as predicted by the $(d, c)$ model. In this figure we plot the drop ratio for each repetition (red points). We also plot the

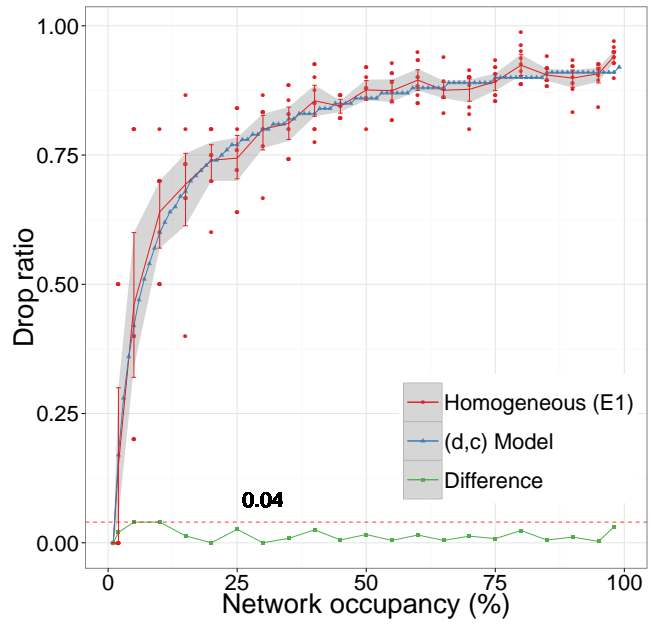

Figure 4: Results for Homogeneous case (E1): we see how close the $(d, c)$ model-predicted values and the simulated values are.

average interpolation up to a $95 \%$ confidence interval envelope (gray area within the error bars). Again, we see how close the simulated and model results are. Indeed, we compute the average case for the 10 repetitions and graph the difference between the average and the model. We see that the maximum difference between both is 0.04 . Of course this is only true for the average case. We will see a bigger difference if we include the variance (points dispersion), especially when the number of sources is small: when we have less sources, the chance of dropping a message is lower, but not zero (bigger variance); when we have more sources, chances of eventually dropping are almost $100 \%$ (smaller variance).

\section{B. Homogeneous case: Anycast}

In simulation $E 2$, we proceed similarly to $E 1$ except that we increase $D$. We set $\lambda=500$ and we increase both the num- 
ber of sources and destinations. Since all destinations are indistinguishable, each time we meet one a message is delivered. Figure 5 (on page 6) shows the evolution of the drop ratio when increasing the number of destinations for different values of $S$ such as $\rho \in\{5 \%, 10 \%, 20 \%, 30 \%, 40 \%, 50 \%, 60 \%, 70 \%\}$. The number of destinations $D$ varies up to the maximum amount of free nodes on each case, i.e. $1 \leq D<N-S$. We can see how adding destinations reduces the drop ratio because of the increase of delivery probability. We see the match between the model and simulated results. We also notice that the variance is lower with a higher number of sources as in the $E 1$ case. This graph allows to define how many nodes are needed in an anycast sensor network to keep the drop ratio bounded. Indeed, we observe that with 20 anycast destinations we can obtain a drop ratio lower than $25 \%$ for a network occupancy in between $30 \%$ and $40 \%$.

\section{Heterogeneity with two classes}

In this section we discuss both simulations $E 3$ and $E 4$ with the main results for the two-class $\left(d, c_{1}, c_{2}\right)$ model. We have $N=100$ nodes, $D=1$ destination, and the rest of nodes divided in two classes $\mathcal{C}_{1}$ and $\mathcal{C}_{2}$ of same size $\left(N_{1}=50\right.$ and $N_{2}=49$ respectively). We increase the number of sources selecting randomly $M_{1}$ from $\mathcal{C}_{1}$ and $M_{2}$ from $\mathcal{C}_{1}$ such that $M=M_{1}+M_{2}$. We define $\lambda_{1}=2.5, \lambda_{2}=200, \lambda=66.6$ for $E 3$ and $\lambda_{1}^{\prime}=10, \lambda_{2}^{\prime}=100, \lambda^{\prime}=\frac{\lambda_{1}^{\prime}+\lambda_{2}^{\prime}}{2}=55$ for $E 4$.

Figure $6 \mathrm{a}$ presents the compared model results for three cases: $(d, c)$ model and $\left(d, c_{1}, c_{2}\right)$ for $E 3$ and $E 4$ simulations. First, we can see that the homogeneous case performs worse than both two-class heterogeneous cases. Recall that the probabilities of the $(d, c)$ model are independent of $\lambda$ while in the $\left(d, c_{1}, c_{2}\right)$ model they are not. Indeed, we see how the assigned values for the $E 3$ case give a lower drop ratio than $E 4$. This is due to the encounter rates defined: on simulation $E 3$, we have $\lambda_{1}<\lambda<\lambda_{2}$. This means that nodes in class $\mathcal{C}_{1}$ meet more frequently than the nodes in $\mathcal{C}_{2}$, and nodes in $\mathcal{C}_{2}$ meet more frequently a node from $\mathcal{C}_{1}$ than from their own class. Consequently, messages in the first class will reach the destination more frequently than the ones in the second class, and messages in the second class can also benefit from the transfer opportunities to the first class to be in turn forwarded to the destination. Since messages are being delivered more frequently, we decrease the drop ratio in comparison with the homogeneous case. On contrast, on simulation $E 4$ the drop ratio increases. If we think in terms of interactions between nodes, the total number of pairs is 4950 , with $\sim 1225$ pairs per group. The number of interactions is distributed as $\mathcal{C}_{1} \sim 25 \%$, $\mathcal{C}_{2} \sim 25 \%$ and $50 \%$ for the inter-class interactions. In $E 4$, the rates are in the same configuration as $E 3$, but with a difference in the order of magnitude as $\lambda_{1}^{\prime}<\lambda^{\prime}<\lambda_{2}^{\prime}$ : values $\lambda_{1}^{\prime}$ and $\lambda_{2}^{\prime}$ in $E 4$ are closer to a unique $\lambda$ value than $\lambda_{1}$ and $\lambda_{2}$ in $E 3$. Hence, more than half of interactions behave similarly in terms of encounter frequency, which explains why $E 4$ is closer to the homogeneous case than $E 3$. We see how the frequency of contacts is key to characterizing the heterogeneity.

Figure 6 shows how the two-class model matches our simulations in both number of drops and drop ratio. In this figure we include the model for both $E 3$ and $E 4$, as well as the simulation results in a $95 \%$ confidence interval. Like in

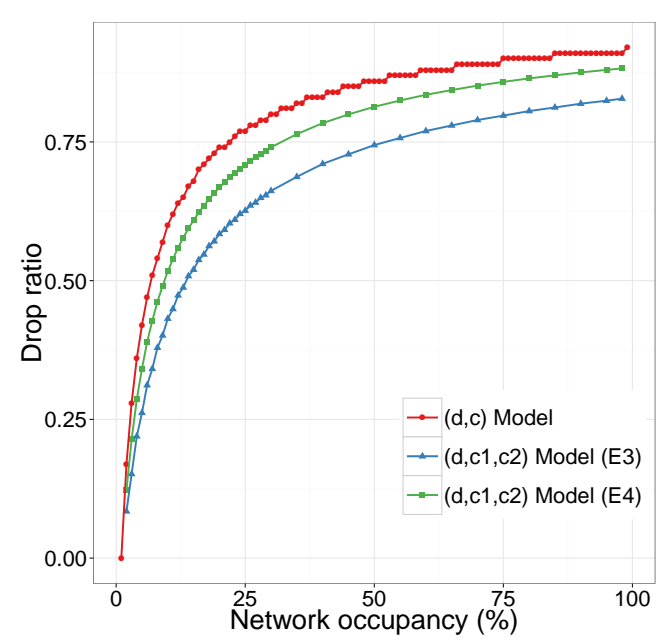

(a) Comparison between the $(d, c)$ and the $\left(d, c_{1}, c_{2}\right)$ models. For the $\left(d, c_{1}, c_{2}\right)$ model, different ICT parameters are considered (E3 and E4).

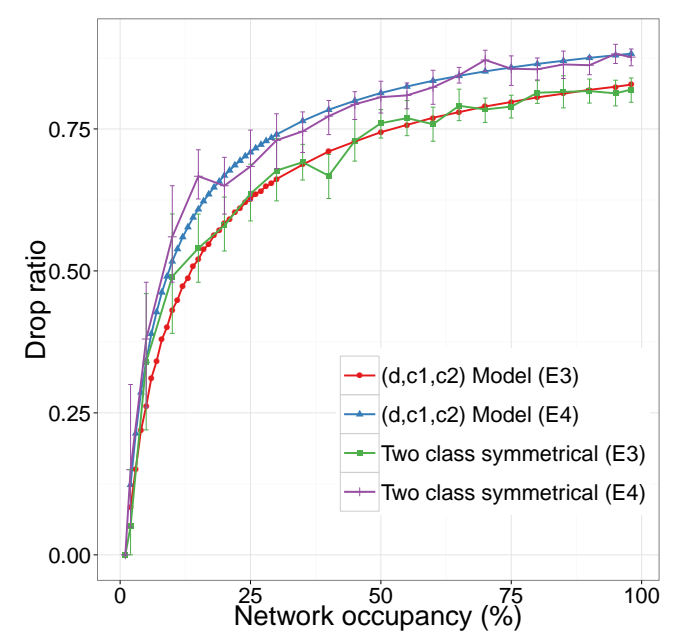

(b) Results for $E 3$ and $E 4$ : we see how close the $\left(d, c_{1}, c_{2}\right)$ model-predicted values and the simulated values are for the symmetrical case.

Figure 6: Drop ratio for two-class simulations $E 3$ and $E 4$.

the homogeneous case, we observe that with less sources we have a bigger variance on the simulated results.

\section{CONCLUSIONS}

In this work, we studied the drop ratio for the progression of messages from a set of sources to a set of destinations. Each source emits one message that can be absorbed by any destination. Messages in our study are simply forwarded among nodes to avoid the inclusion of extra copies (replication), which will only increase the probability of drops. We worked with nodes with 1-message buffers to represent the worst case: all nodes are saturated and we want to know how many new messages can be injected (upper bound). Our main contribution is the introduction of a continuous time Markov chain model to characterize the drop of messages under these hypotheses. We introduced two variants: the $(d, c)$ model for 

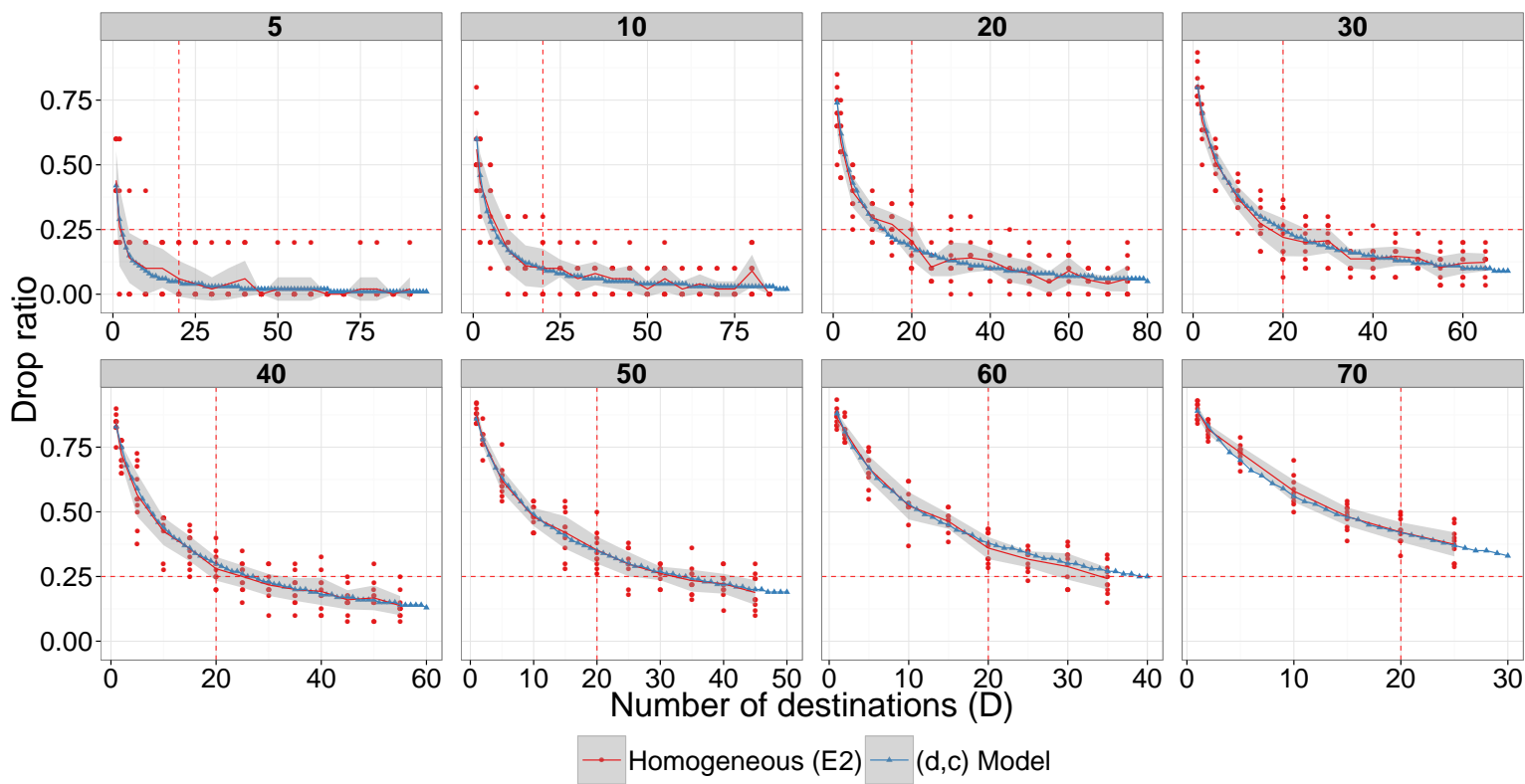

Figure 5: Results for E2: each graph represents a fixed network occupancy $\rho$. We see how adding destinations decreases the drop ratio as predicted by the model. We see the number of destinations needed to get a $25 \%$ drop ratio for each case.

homogeneous contact between nodes and the $\left(d, c_{1}, c_{2}\right)$ for a two-class heterogeneous case. Based on these models, we show the link between the encounter rate among nodes with the drop ratio of forwarded messages: the selection of these rates can impact the message dropping behavior. In specific, we showed that some configurations behave better than the homogeneous case, while others behave close to it. From the model we can extract the trade-off between the number of sources and destinations nodes in order to achieve a desired drop ratio requirement. We performed a DTN simulation to calculate the drop ratio for several scenarios to validate the model. We showed that the outputs of the CTMC model fit to DTN simulation outputs. For future work, we plan to better characterize the behavior of the two-class model by varying encounter frequencies. We will also investigate the cases of larger buffers and full heterogeneity.

\section{ACKNOWLEDGMENTS}

The authors would like to thank Patrick Sénac for his ideas to improve this work. This work is partially supported by CONICYT (Becas Chile PhD program).

\section{REFERENCES}

[1] K. Fall, "A delay-tolerant network architecture for challenged internets," ser. ACM SIGCOMM 2003, pp. 27-34.

[2] M. Conti and S. Giordano, "Mobile ad hoc networking: milestones, challenges, and new research directions," IEEE Communications Magazine, vol. 52, no. 1, pp. 85-96, January 2014.

[3] R. Groenevelt, P. Nain, and G. Koole, "The message delay in mobile ad hoc networks," Performance Evaluation, pp. 210-228, 2005.

[4] Z. Haas and T. Small, "A new networking model for biological applications of ad hoc sensor networks," IEEE/ACM Trans. on Networking, vol. 14 , no. 1 , pp. $27-40,2006$.

[5] Y.-K. Ip, W.-C. Lau, and O.-C. Yue, "Performance modeling of epidemic routing with heterogeneous node types," in IEEE ICC 2008, pp. 219-224.
[6] A. Hanbali, P. Nain, and E. Altman, "Performance of ad hoc networks with two-hop relay routing and limited packet lifetime," in IEEE IWQoS 2006, pp. 295-296.

[7] M. Abdulla and R. Simon, "The impact of the mobility model on delay tolerant networking performance analysis," in ANSS 2007, pp. 177-184.

[8] T. Spyropoulos, K. Psounis, and C. Raghavendra, "Efficient routing in intermittently connected mobile networks: The multiple-copy case," IEEE/ACM Trans. on Networking, 2008.

[9] R. Diana and E. Lochin, "Modelling the delay distribution of binary spray and wait routing protocol," in IEEE WoWMoM 2012.

[10] X. Zhang, G. Neglia, J. Kurose, and D. Towsley, "Performance modeling of epidemic routing," Computer Networks, vol. 51, no. 10, pp. 2867-2891, 2007.

[11] L. Sassatelli and M. Medard, "Inter-session network coding in delaytolerant networks under spray-and-wait routing," in WiOpt 2012.

[12] R. Gunasekaran, V. Mahendran, and C. Siva Ram Murthy, "Performance modeling of delay tolerant network routing via queueing petri nets," in IEEE WoWMoM 2012.

[13] F. Brauer and C. Castillo-Chavez, "Epidemic models," in Mathematical Models in Population Biology and Epidemiology, ser. Texts in Applied Mathematics. Springer, 2012, pp. 345-409.

[14] T. Spyropoulos, T. Turletti, and K. Obraczka, "Routing in delay-tolerant networks comprising heterogeneous node populations," IEEE Trans. on Mobile Computing, vol. 8, no. 8, pp. 1132-1147, 2009.

[15] C. Boldrini, M. Conti, and A. Passarella, "Modelling social-aware forwarding in opportunistic networks," in Performance Evaluation of Computer and Communication Systems. Milestones and Future Challenges, ser. Lecture Notes in Computer Science. Springer, 2011, vol. 6821, pp. 141-152.

[16] A. Picu, T. Spyropoulos, and T. Hossmann, "An analysis of the information spreading delay in heterogeneous mobility DTNs," in IEEE WoWMoM 2012

[17] V. Manam, V. Mahendran, and C. Siva Ram Murthy, "Performance modeling of routing in delay-tolerant networks with node heterogeneity," in COMSNETS 2012, pp. 1-10.

[18] R. Subramanian and F. Fekri, "Throughput analysis of delay tolerant networks with finite buffers," in IEEE MASS 2008. 